\title{
RESPONSES OF CAT AUDITORY NERVE FIBERS TO BIPHASIC ELECTRICAL CURRENT PULSES
}

\author{
E. Javel, PhD; Y. C. Tong, BE, PhD; R. K. Shepherd, BSc, PhD; G. M. Clark, PhD, FRaCS
}

This work was supported in part by a grant from the National Institute of Neurological and Communicative Disorders and Stroke awarded to E. Javel.

Discharge patterns of single auditory nerve fibers were recorded from normal-hearing cats implanted with a 12-band intracochlear electrode array. Stimuli were biphasic current pulses of specifiable width, amplitude, and rate. Acoustic tuning curves were obtained to determine the cochlear positions of the fibers. Response latencies to electrical stimuli formed two groups. Short latency (0.3 to $0.7 \mathrm{~ms}$ ) responses were attributed to direct activation of spiral ganglion neurons. At high stimulus intensities, these often exhibited abrupt shifts toward even shorter latencies. Long latency (>1.5 ms) responses were probably caused by electrophonic activation of functional hair cells. Response thresholds to electrical stimuli depended on a fiber's proximity to the stimulating electrodes, and they did not depend on a fiber's acoustic response threshold or spontaneous discharge rate. High intensity $(>1.5 \mathrm{~mA})$ stimuli could excite fibers over a wide range of characteristic frequencies, even for the narrowest $(0.45 \mathrm{~mm})$ electrode separations. Response threshold was an exponentially decreasing function of pulse width for widths up to $300 \mu \mathrm{s} /$ phase. Fiber discharges were highly phase-locked at all suprathreshold intensities, and saturation discharge rates usually equaled stimulus pulse rates for rates up to at least 800 pulses/s. Dynamic ranges were small (1 to $6 \mathrm{~dB}$ ), increased with pulse rate, and were uncorrelated with electrical response threshold. Within the dynamic range, shapes of poststimulus time and interspike interval histograms resembled those obtained in response to acoustic stimuli. Depolarization block caused fiber activity to cease in 2 to 5 seconds for sustained stimuli presented at high ( $>600 \mathrm{pulses} / \mathrm{s}$ ) pulse rates and intensities.

It is of considerable importance that neural responses to both electrical stimuli and speech stimuli be understood thoroughly if the speech-processing strategies used in contemporary cochlear implants are to continue their evolution. Not only are the physiological data necessary to the evaluation of speech-processing strategies, but they also allow new psychophysical experiments to be designed and provide the basis for interpreting behavioral findings.

Previous studies ${ }^{1-6}$ have presented various types of electrical waveforms through several different kinds of intracochlear and extracochlear stimulating electrodes. These studies have shown that electrical stimuli can elicit stimulus-locked discharges from auditory nerve fibers and that many of the temporal features of stimulus waveforms are retained in the responses of cells in more central nuclei. They have also demonstrated that neuronal response latency can vary, that neuronal discharges are highly synchronized or phase-locked to the stimulus waveform, and that phase-locked activity persists to higher stimulus frequencies than occurs in response to acoustic tones. In addition, they have shown that high intensity stimuli can excite neurons over a wide range of characteristic frequencies, that the shapes of "tuning curves" for sinusoidal electrical stimuli are invariant across cells, and that stimulus-evoked discharge rates are rapidly increasing functions of the stimulus current.

The studies described here were undertaken to determine some of the fundamental response properties of auditory nerve fibers to electrical stimuli presented through a banded electrode array placed in the scala tympani. Our major interest was in characterizing the fine and gross time structures and input-output relationships of auditory nerve fiber response to the electrode arrays and stimuli used in the Melbourne cochlear implant. ${ }^{7,8}$ Our purpose was to provide data that could be used to explain behavioral findings in humans implanted with a similar device and to examine the performance limits of the device.

\section{METHODS}

Adult cats with normal hearing and no evidence of ex- ternal or middle ear disease were anesthetized with sodium pentobarbital $(40 \mathrm{mg} / \mathrm{kg}$ ) and mounted in a rigid headholder. Supplemental anesthesia was given as necessary throughout the experiment. Core temperature was held at $37^{\circ} \mathrm{C}$ using a DC heating pad.

After the bulla was opened and the round window membrane reflected, a custom-built intracochlear electrode array was inserted into the scala tympani for a distance of approximately $6 \mathrm{~mm}$. The electrode array possessed 12 circumferential platinum bands, each having a width of $0.3 \mathrm{~mm}$. The bands were positioned on a silicone rubber carrier at $0.75-\mathrm{mm}$ centers. Once the electrode array had been installed, cyanoacrylate cement and polyester mesh were used to fix it in position.

The procedures used to expose the auditory nerve and record from single fibers were the same as those described by Javel.' Acoustic stimuli were synthesized digitally and presented by a condenser microphone through a calibrated distortion-compensating network and custom-made hollow speculum.

Electrical stimuli were biphasic charge-balanced current pulses delivered by an optically isolated current source. Pulse duration, amplitude, and repetition rate could be set under computer control, and the electrodes used to source and sink the current could be selected manually. The data presented here were obtained using the most apical electrode band as the current source and the adjacent band as the current sink.

\section{RESULTS AND DISCUSSION}

Following implantation, auditory nerve fibers initially exhibited normal-appearing spontaneous activity, acoustic tuning curves, and input-output functions. However, fiber responses typically deteriorated as experiments proceeded past the first day. That is, although spontaneous activity was maintained, acoustic response thresholds became elevated and tuning curves were tuned less sharply. Spontaneous activity and responses to acoustic stimuli were gradually lost as experiments proceeded past the second day. 

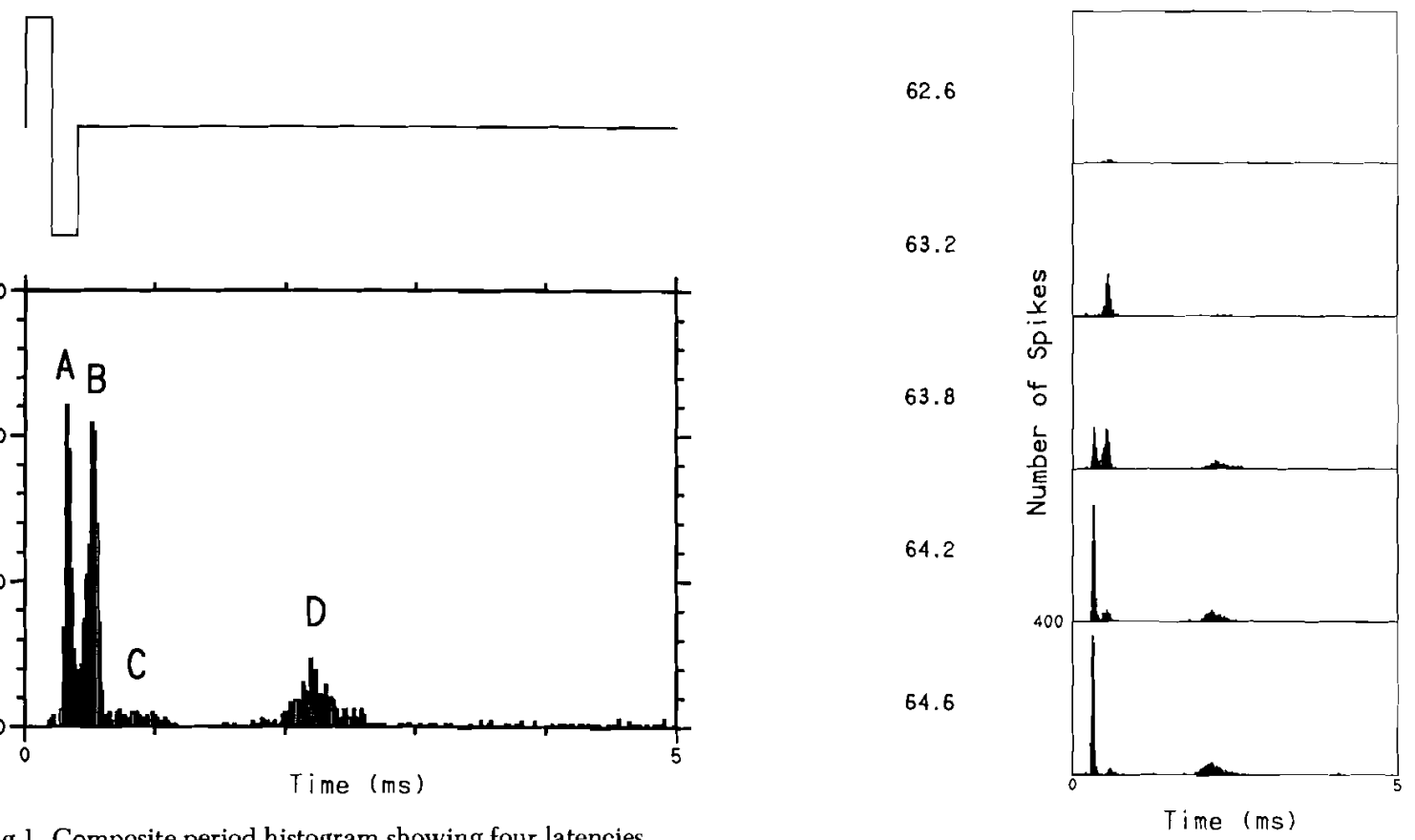

Fig 1. Composite period histogram showing four latencies, labeled A to D, at which auditory nerve fibers could discharge in response to biphasic electrical current pulse. Stimulus waveform is shown at top.

A schematic representation of an auditory nerve fiber's response to a biphasic current pulse is shown in Fig 1. At the top is shown one period of the electrical stimulus, and at the bottom is shown the time structure of the response. The response has four distinct components, which we have labeled as A, B, C, and D, based on their times of occurrence relative to the electrical pulse. Very rarely did fiber responses show all four components simultaneously.

The A and $\mathrm{B}$ responses are highly synchronized to the current pulse, and they occur at latencies of about 0.3 and

Fig 3. Poststimulus time (PST) and interspike interval (ISI) histograms obtained at various intensities in response to $200-\mu \mathrm{s} /$ phase pulse trains presented at 200 pulses $/ \mathrm{s}$ for $100 \mathrm{~ms}$. Intensities are in decibels re $1 \mu \mathrm{A}$.

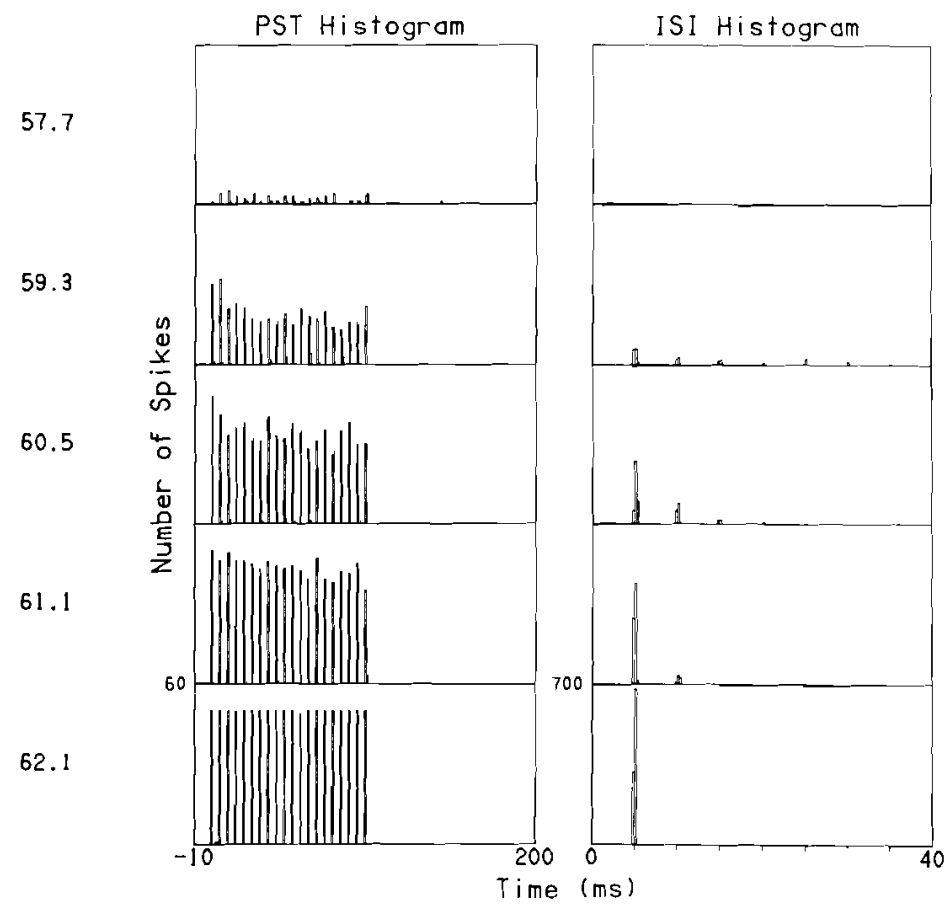
The A response tended to possess a higher threshold than
the B response; it usually grew more rapidly with increasing intensity, and it was also more tightly synchronized. In addition, the width of the $B$ response obtained at nearthreshold intensities depended on electrical pulse dura-

$0.6 \mathrm{~ms}$ after pulse onset. Using monopolar stimuli, van den Honert and Stypulkowski ${ }^{4}$ have suggested that these responses are generated at the cell body and peripheral dendrite, respectively, of the spiral ganglion neuron.

Fig 2. Period histograms obtained in response to $200-\mu s$ phase pulses presented at five different intensities. Intensiphase pulses presented at
ties are in decibels re $1 \mu \mathrm{A}$ 
tion, with wider pulses producing greater temporal dispersion of the B peak. However, even though the width of the $B$ response could vary at near-threshold intensities, the $B$ response was always highly synchronized at intensities that were well above threshold. The $\mathrm{C}$ response is a diffuse group of spikes occurring at latencies of 0.7 to $1.2 \mathrm{~ms}$. It did not always appear, was relatively small in size, and occurred only near the electrical response threshold. The D responses, when they occurred at all, arose at latencies of 1.5 to $2.5 \mathrm{~ms}$. As Moxon ${ }^{2}$ has noted, the long latency implies that the $\mathrm{D}$ response probably is not a direct consequence of the electrical activation of the cell. Because the $\mathrm{D}$ response is indistinguishable from the response evoked by an acoustic click, it is likely that it arises as a result of electrophonic activation of hair cells.

The most commonly observed progression of these responses with increasing intensity is shown in Fig 2, which depicts period histograms, that is, plots of spike density within the electrical stimulus period. Only the $B$ response occurred at threshold, and its latency was 0.6 to $0.7 \mathrm{~ms}$. The $B$ response increased in size as intensity increased and as the electrical pulses became more effective in eliciting discharges from the fiber. No $\mathrm{C}$ response was apparent in these data, but a $\mathrm{D}$ response arose at suprathreshold levels. As intensity increased, a point was ultimately reached where a shorter latency $A$ response appeared. The $A$ response was very highly synchronized to the stimulus, and it grew rapidly at higher intensities at the expense of the $B$ response. At the highest intensities (not shown) the $B$ response disappeared entirely, leaving only the $A$ response and the $D$ response. Although this progression was the one we observed most often, it was not the only one we saw. With the exception of the abrupt conversion of a $B$ response into an A response, these data are identical to those described earlier by van den Honert and Stypulkowski. ${ }^{4}$ The mechanism underlying the conversion of a $B$ response into an $\mathrm{A}$ response requires further investigation. It is likely that electrode geometry and stimulus waveform are important parameters affecting the behavior of these two short latency responses.

We found that electrical response thresholds depended on the proximity of the fiber's cochlear position to the stimulating electrodes, on the spatial separation between stimulating electrodes, and on the electrical pulse width. The closer a fiber was to the stimulating electrodes, the lower was its threshold. The lowest thresholds we encountered for pulse widths of $200 \mu \mathrm{s} /$ phase were 0.2 to $0.3 \mathrm{~mA}$, and these occurred only for fibers whose characteristic frequencies were above $10 \mathrm{kHz}$. Also, wider separations between source and sink electrodes led to lower electrical response thresholds for most fibers, as did longer pulse widths.

Fibers spatially distant from the stimulating electrodes could usually be excited at fairly high stimulus currents, even though current is ostensibly being passed only between two narrowly spaced electrode bands. Although these thresholds always exceeded $1 \mathrm{~mA}$, their existence can be taken as an indication that spatial localization of activity is lost at high current levels, even for narrow electrode spacings. It also means that substantial interactions between stimuli delivered to nearby channels can be expected at high current levels.

Poststimulus time (PST) and interspike interval (ISI) histograms of responses to electrical pulse trains presented at 200/s are shown in Fig 3. A feature of activity elicited
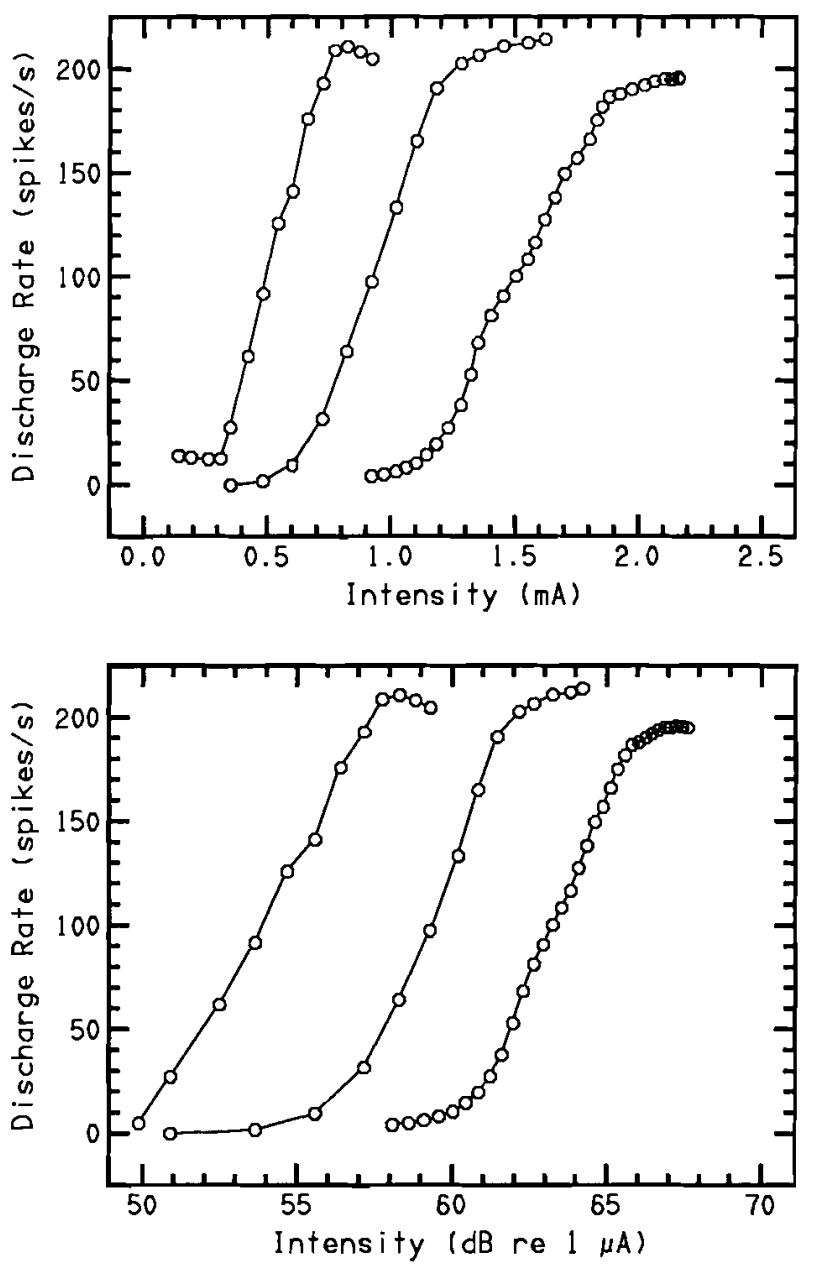

Fig 4. Rate-intensity functions for three fibers in response to $200-\mu \mathrm{s} /$ phase pulses presented at $200 \mathrm{~Hz}$ in 100 -ms bursts. Top - rate-intensity curves expressed as linear function of stimulus current. Bottom - same data displayed as logarithmic (decibel) function of current.

by the electrical stimulus is that it simply adds to the fiber's spontaneous activity. This is not evident in Fig 3, however, because that particular fiber exhibited no spontaneous activity.

Within the dynamic range of the response, the structure of PST histograms obtained for electrical stimuli was not much different from those obtained for acoustic tones. In contrast with what is generally assumed, this finding suggests that adaptation in auditory nerve fiber responses has a component that is apparently contributed by the spiral ganglion cells, and that adaptation is not determined solely by properties of the hair cell/auditory nerve synapse.

There were three differences between the gross time structure of responses to electrical pulses and responses to acoustic tones. First, at sufficiently high intensities every electrical pulse became capable of eliciting a spike, producing a rectangular PST histogram that has no counterpart in responses to acoustic tones. Second, spontaneous activity (if present) is not abolished when the electrical stimulus ends. And third, fibers could cease to respond to electrical stimuli presented at high $(>600 / \mathrm{s})$ pulse rates and intensities. This took 2 to 5 seconds to develop, depending on the intensity and pulse rate, and it was accompanied by the shrinkage and ultimate disappearance of the 


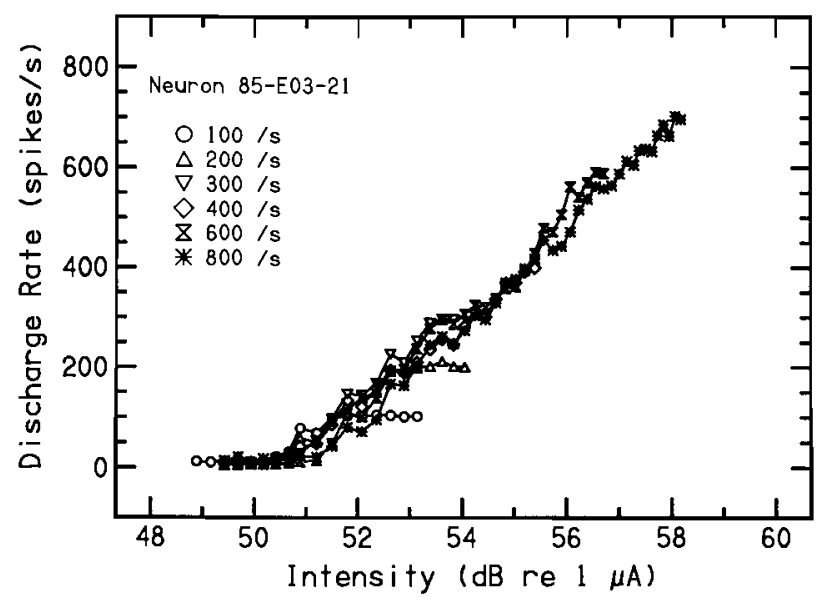

Fig 5. Rate-intensity functions obtained from auditory nerve fiber in response to $200-\mu$ s/phase pulse trains presented at indicated pulse rates.

fiber's action potential. The loss of response is almost certainly the result of depolarization block, that is, an inability of the cell membrane to repolarize in time to conduct the next action potential.

Another feature of fiber response shown in Fig 3 is that of synchronization. Recall that for acoustic stimuli, a tone presented at a low enough frequency will cause discharges in the auditory nerve to become partially entrained or time-locked to the stimulus period. In terms of ISI, the entrainment means that certain time intervals will occur more often than others and that intervals between successive spikes will tend to group around integer multiples of the stimulus period.

The basic structure and progression of ISI histograms obtained in response to electrical pulse trains were similar to those obtained in response to acoustic tones, except that 1) electrically evoked spikes are synchronized to a considerably greater degree than acoustically evoked spikes, 2) the degree of synchronization exceeds anything observed electrophysiologically in "acoustic" hearing, and 3) at high intensities all of the ISIs occur at times corresponding to the period of the electrical stimulus, even for pulse rates in excess of 800 pulses/s.

Representative input-output functions for discharge rate are shown in Fig 4 for three different fibers. One of the fibers possessed a low response threshold to electrical stimuli, one had a medium threshold, and one had a high threshold. The stimulus in all three cases was a pulse train presented at a rate of $200 / \mathrm{s}$ with a pulse width of $200 \mu \mathrm{s} /$ phase. In all three cases the response saturated or reached a maximum at the pulse rate, and the only major difference between the curves was response threshold.

Consistent with the findings of other investigators, ${ }^{2-5}$ we observed that the range of intensities over which response magnitude increases (the dynamic range) is rather small. Dynamic ranges in our sample extended from a low of 0.9 $\mathrm{dB}$ to a high of $6.1 \mathrm{~dB}$. There was no obvious relationship between dynamic range and electrical response threshold, and the distribution of dynamic range for biphasic pulses was the same for all fibers but the ones with the highest thresholds. That is, high threshold fibers tended to possess narrower dynamic ranges.

Input-output functions for discharge synchronization

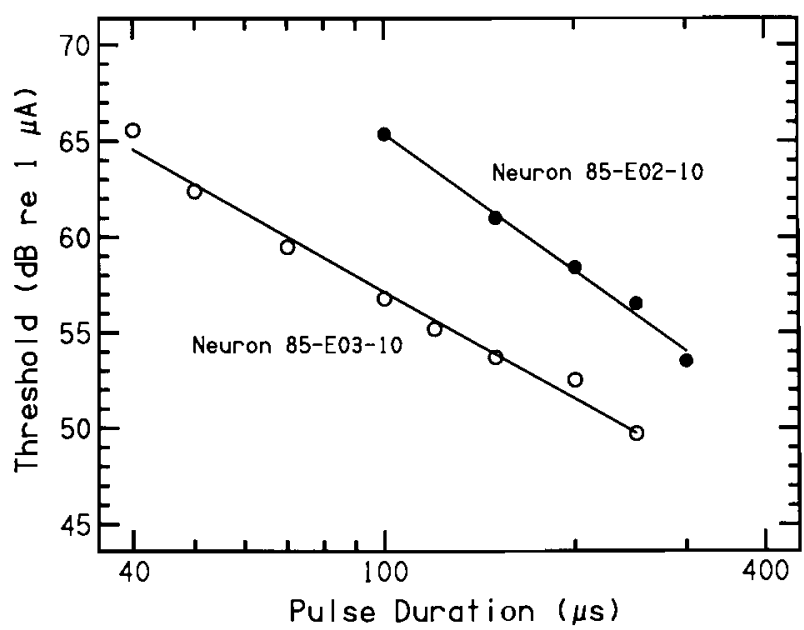

Fig 6. Effect of electrical pulse duration on electrical response threshold for two fibers. Pulse durations indicated are for each phase of biphasic pulse. Solid lines indicate linear regression fits to data.

were very steep, with maximum synchronization usually being attained within $1 \mathrm{~dB}$ of electrical response threshold.

We found that fiber dynamic range for discharge rate was a function of both electrical pulse rate and response threshold. Figure 5 shows several input-output functions, each of which was obtained for pulse trains presented at rates that ranged from 100 to 800 pulses/s. The six curves in this Figure virtually overlie one another, which implies that 1) there are no differences in rates of growth of discharge rate with increasing intensity for electrical stimuli presented at different pulse rates, and 2) eliciting a given discharge rate depends only on stimulus current and not on pulse rate. For biphasic pulses, it is likely that dynamic range is governed by two processes, namely the buildup of stimulus current and neural refractoriness.

One other notable point in Fig $\mathbf{5}$ is that although dynamic ranges are very limited, intensity coding within the dynamic range is extremely accurate. This suggests that linear compression techniques should be successful in expanding dynamic range upward to values that are more useful than 1 to $6 \mathrm{~dB}$.

Representative data on the relationship between electrical response threshold and current pulse width are shown in Fig 6, where response threshold in decibels for two different fibers has been plotted as a function of the duration of each pulse phase. One fiber had a fairly low threshold to electrical pulses, and the other had a high threshold. The data values were obtained by using a weighted least squares procedure to fit input-output curves with a saturating power function. Threshold, defined as the intensity producing a discharge rate $10 \%$ above the spontaneous rate, was estimated from each fit, and these are shown in Fig 6 by the open and filled symbols. The solid lines represent the results of linear regressions performed on these data. It is apparent that electrical response threshold is a simple decreasing function of pulse width, for pulse widths up to at least $300 \mu \mathrm{s} /$ phase, and that there is almost no difference in response behavior of fibers with different thresholds.

The data shown here support the notion that the suprathreshold behavior of auditory nerve fiber responses to electrical stimuli is exceptionally uniform. This uniformity 
suggests that modeling of these responses should produce very acceptable results.

\section{REFERENCES}

1. Moxon EC. Electrical stimulation of the cat's cochlea: a study of discharge rates in single auditory nerve fibers [MSEE thesis]. Cambridge, Mass: Massachusetts Institute of Technology, 1965.

2. Moxon EC. Neural and mechanical responses to electric stimulation of the cat's inner ear [Dissertation]. Cambridge, Mass: Massachusetts Institute of Technology, 1971.

3. Hartmann R, Topp G, Klinke R. Electrical stimulation of the cat cochlea - discharge pattern of single auditory fibres. Adv Audiol 1984;1:18-29.

4. Van den Honert C, Stypulkowski PH. Physiological properties of the electrically stimulated auditory nerve. II. Single fiber re- cordings. Hear Res 1984;14:225-43.

5. Clopton BM, Glass I. Unit responses at cochlear nucleus to electrical stimulation through a cochlear prosthesis. Hear Res 1984;14:1-11.

6. Merzenich MM, Michelson RP, Pettit CR, Schindler RA, Reid M. Neural encoding of sound sensation evoked by electrical stimulation of the acoustic nerve. Ann Otol Rhinol Laryngol 1973;82: 486-503.

7. Clark GM, Tong YC. A multiple-channel cochlear implant. Arch Otolaryngol 1982;108:214-8.

8. Tong YC, Clark GM, Blamey PJ, Busby PA, Dowell RC. Psychophysical studies for two multiple-channel cochlear implant patients. J Acoust Soc Am 1982;71:153-60.

9. Javel E. Suppression of auditory nerve responses. I. Temporal analysis, intensity effects and suppression contours. J Acoust Soc Am 1981;69: 1735-45. 


\section{University Library}

\section{- M M N E R VA A gateway to Melbourne's research publications}

Minerva Access is the Institutional Repository of The University of Melbourne

Author/s:

Javel, E.;Tong, Y. C.;Shepherd, R. K.;Clark, Graeme M.

Title:

Responses of cat auditory nerve fibers to biphasic electrical current pulses

Date:

1987

Citation:

Javel, E., Tong, Y. C., Shepherd, R. K., \& Clark, G. M. (1987). Responses of cat auditory nerve fibers to biphasic electrical current pulses. Annals of Otology, Rhinology \& Laryngology, January-February, 96(1, part 2, suppl.128), 26-30.

Persistent Link:

http://hdl.handle.net/11343/27252 